\title{
2 Vorbemerkungen zur Architektur von Schulhäusern
}

Die Erstellung von Schulgebäuden ist ein komplexes Unterfangen, in das unterschiedliche staatliche und nichtstaatliche Akteure eingebunden sind. Typischerweise entstehen Schulgebäude in Wellen der Bildungs- und/oder Bevölkerungsexpansionen. Ihre Architekturen werden geprägt durch Fragen des Auftrags der Gebäude: Für welche Unterrichtsformen und welche Vorstellungen, guten Unterrichts' wurden sie gebaut? Gleichzeitig erhebt sich die Frage ihrer Repräsentation: Wie stellen sich Schule und Gemeinde als Bauherrschaft dar? Solche Fragen prägen wesentlich den Möglichkeitsraum, in welchem Schulgebäude entstehen. Sie sollen im Folgenden skizziert werden.

\subsection{Zur Geschichte des Schulhausbaus}

Die Entstehung der ersten Schulbauten öffentlich-rechtlicher Schulen geht mit der Genese des staatlichen Volksschulwesens und damit mit der Genese der bürgerlich-demokratischen Nationalstaaten einher. Letztere sind eng mit den politischen Wirren, Umbrüchen und Instabilitäten des ausgehenden 18. Jahrhunderts und der ersten Hälfte des 19. Jahrhunderts verbunden. Die zunehmende Industrialisierung und die Nationalstaatsbildungen sind wechselseitig mit einem tiefschürfenden gesellschaftlichen, politischen und soziostrukturellen Wandel verschränkt. Einer der Dreh- und Angelpunkte der politischen Konflikte zwischen Kirche und konservativ-katholischen Gruppierungen einerseits und den liberalen Kreisen anderseits ist die Schaffung eines öffentlich-rechtlichen Schulwesens (siehe Criblez et al. 1999). Die Schule wird zu einer politischen Streitfrage, da sie als institutionalisierte Erziehungs- und Bildungsinstanz sowohl das Verhältnis zwischen Staat und Bürger entscheidend strukturiert als auch tief in die Lebensweisen der Bürger_innen eingreift.

Ein erster Versuch, eine staatliche, liberale Bildungspolitik zu schaffen, endet mit dem Scheitern der Helvetik (1798-1803). Mit den liberalen Verfassungen, die sich ein Teil der Kantone um 1830 geben, setzen umfassende Reformen ein. Dabei ist die Schaffung einer Volksschule eine der zentralen revolutionären Forderungen, die zu einer der wichtigsten Staatsaufgaben der jungen Demokra- 
tien wird. Sie ist unabdingbar für die Erziehung der bis dahin leibeigenen Subjekte zu mündigen Staatsbürgern. Das Schulwesen ist aber auch ein Mittel, Staatlichkeit bis in die entlegenen Gebiete durchzusetzen. Da die Volksschule auf viele Widerstände stößt, gelingt die Schulbildung für alle nur über die Schulpflicht.

Zur Durchsetzung des staatlichen Bildungsmonopols bedarf es einer flächendeckenden Schulinfrastruktur. In den Städten werden in der Regel vorhandene Gebäude zu Schulhäusern umfunktioniert. In den ländlichen Gebieten müssen Schulhäuser meist neu erbaut werden. Die Lehrpersonen lassen sich nicht aus der örtlichen Bevölkerung rekrutieren. Daher wird nicht nur Schulraum, sondern auch Wohnraum benötigt und meist auch weitere Infrastrukturen wie Werkstätten und/oder landwirtschaftliche Gebäude für den Nebenerwerb der Lehrpersonen. Die Schulhäuser sind quasi die ersten Bauten der jungen liberaldemokratischen Staaten, die bis in die ländlichen und damit tendenziell konservativen Regionen reichen und den Staat dort materialisieren.

Die große Bautätigkeit in der flächendeckenden Erstellung von Schulhäusern stellt damit jenseits der schulischen Funktion der Gebäude auch eine Manifestation des jungen Nationalstaates dar. Denn architektursoziologisch betrachtet ist eine zentrale Funktion gebauter Strukturen der Erkennungs- und Orientierungswert, den Bauten realisieren. Der Raum und namentlich die Architektur fixieren soziale Sinnstrukturen und schreiben sie einem Ort ein. Simmel (1908: 473) spricht von Raum als einem ,stabilen Drehpunkt labiler Verhältnisse und Wechselwirkungen“. Dabei ist die Architektur vor allem in ihrer Symbolhaftigkeit gemeint. Als Symbole stehen Gebäude für Bedeutungsstrukturen, welche „die Wirklichkeit der Alltagswelt überschreiten“, wie etwa „ein abstraktes moralisches Prinzip, eine kollektive Idee oder gar die Anwesenheit von etwas „Heiligem““ (Steets 2015: 192, Hervorhebung im Original). Das Schulhaus kann in dieser Perspektive nicht nur als neues Zentrum in den Dörfern gesehen werden. Die Schulbauten sind zugleich Sinnbilder und Werkzeuge der Durchsetzung einer neuen politischen und sozialen Ordnung.

Dabei ist hervorzuheben, dass sich die Schulhäuser, die vor 1850 erbaut wurden, von Wohnhäusern kaum unterscheiden lassen. Dies wird in der (Forschungs-)Literatur immer wieder hervorgehoben. Die meisten Untersuchungen zum Thema Architektur von Schulbauten setzen daher erst um 1850 ein, mit der Begründung, dass sich erst dann ein eigener Gebäudetyp Schulhaus herausgebil- 
det hat (so beispielsweise Dangel \& Zangger 2008; Gurtner 1997; Helfenberger 2016; Oberhänsli 1996; Schneeberger 2005). Mit dem Schulhaus Grün in Schmitten wird in Kap 7.3. ein Gebäude analysiert, das vor der Ausdifferenzierung eines eigenen Gebäudetypus Schule erbaut wurde. Die Analyse zeigt, dass Schulhäuser, die als Wohngebäude gebaut wurden, nicht nur interpretationsbedürftig sind, sondern auch ergiebige Ergebnisse bezüglich der Konstituierung des jungen liberalen Nationalstaates und der gesellschaftlichen Umwälzungen erlauben. Vorgreifend kann festgehalten werden, dass der Staat sich als urbaner Bauherr gibt, der sich an städtischer Lebensart und arbeitsteiliger, kapitalistischer Ökonomie orientiert. Der Bau von Schulgebäuden verändert daher nicht nur die Dorfbilder. Schulhäuser verkörpern in ihrer Funktion als Wohnhaus und in ihren Repräsentationen auch die neue Gesellschaftsordnung und die kapitalistisch organisierte Ökonomie. Sie sind ein Abbild einer politischen Ordnung in der Schaffung und Trennung von Öffentlichkeit und Privatheit, was auch das Verhältnis zwischen Staat und Bürger mitbetrifft. Schulräume stellen aber auch eine sinnbildlich verkörperte neue Raumorganisation kapitalistischer Produktion her, was sich in der Trennung von Arbeit und Wohnen manifestiert (vgl. Kap. 7.3).

Ab Mitte des 19. Jahrhunderts bildet sich ein relativ einheitlicher Gebäudetypus Schule heraus (vgl. Helfenberger 2013). Die Richtlinien des Kantons Zürich, die von vielen Kantonen als sogenannte ,Normalien“ übernommen wurden, sehen ,einen klassizistischen Baukörper [vor], mit symmetrischen Fassaden, Mittelrisalit und Dreieckgiebel, bisweilen mit Säulenportikus und Kranzgesims, [dieser] wurde zum gängigen Bautypus, der in fast allen Quartieren der Stadt und in den meisten Gemeinden anzutreffen ist" (Dangel \& Zangger 2008: 7). In der Regel sind es Korridorschulhäuser mit zwei oder vier großen Klassenzimmern pro Etage. Sie sind als Prunkbauten konzipiert, die vor allem einer Repräsentationsarchitektur verpflichtet sind und die vom Volksmund Schulschlösser oder Schulkasernen genannt werden. Zu Beginn des 19. Jahrhunderts ist zumindest im Diskurs eine teilweise Abwendung von solchen Prunkbauten festzustellen, obwohl diese bis weit in die Mitte des 20. Jahrhunderts hinein immerhin für die Sekundar- und Mittelschulen den Maßstab setzten. In der Diskussion ist eine Hinwendung zu einer sogenannten kindgerechten Architektur festzustellen, die sich insbesondere in kleinräumigeren Schulbauten und Pavillonanlagen manifestiert. Die kasernen- oder palastartigen Prunkbauten, in ihren spezifischen Raumorganisationen und Positionierungen, stehen jedoch maßgeblich für den Gebäu- 
detypus und damit auch für die Institution Schule, wie dies im empirischen Teil ausführlich thematisiert werden wird. Den Analyseergebnissen soll hier jedoch nicht vorgegriffen werden.

\subsection{Planung und Bau von Schulhäusern}

Der Bau von Schulhäusern ist ein komplexes Unterfangen, das Bedarfsplanung, Standortsuche, Planung, Regulierung und Beaufsichtigung umfasst. Historisch gesehen sind die Planungs- und Bauprozesse im Spannungsfeld eines spezifischen Expertenwissens und der oft durch Laien durchgeführten öffentlichen Kontrolle anzusiedeln (Helfenberger 2016). Dementsprechend sind zahlreiche staatliche und nichtstaatliche Akteure beteiligt.

Im subsidiär aufgebauten politischen System der Schweiz sind auf staatlicher Seite verschiedene Departemente der drei Verwaltungsebenen - Bund, Kantone und Gemeinden - involviert. Verfassungsmäßige Rechte schreiben der Gemeindeautonomie zu, „Teile der öffentlichen Angelegenheiten eigenverantwortlich zu gestalten. Im Schulwesen kommt diesem Recht eine zentrale Bedeutung $\mathrm{zu}$, weil der staatliche Bildungsauftrag maßgeblich durch die Gemeinden umgesetzt wird“ (K. Meyer 2016: 55). So sind die Gemeinden Träger der Primarschulen. Sie haben das Hausrecht inne und sind damit aber auch für die Finanzierung $^{2}$, Planung, Erstellung und den Unterhalt der Schulanlagen zuständig. Auch die Bedarfsplanung und Standortsuche sind kommunale Aufgaben. $\mathrm{Zu}$ den Schulanlagen gehört, dass die Gemeinden geeignete Sportanlagen für den Turnunterricht bereitstellen müssen.

Die mit Planung und Bau der Schulhäuser beauftragten kommunalen Akteure sind in der Regel in den Baudepartementen angesiedelt. Die Erziehungs- und Bildungsdepartemente verfügen nicht selbst über die Bauaufgaben. Infrastrukturplanung und -unterhalt der Schule sind in allen mir bekannten Gemeinden in anderen Departementen verortet als der Schulbetrieb, ohne dass eine Mitwirkung

2 Meines Wissens gab es in den meisten Kantonen einen Fonds, der armen Gemeinden bei der Finanzierung von Schulbauten behilflich war. Die kantonalen Zuschüsse wurden ab 1950 aufgelöst. 
oder Partizipation seitens der Schule vorgesehen ist, wie dies in Deutschland teilweise der Fall ist (Montag Stiftung Jugend und Gesellschaft Bonn 2011). Die Schweizer Schulen verfügen über keine Autonomie oder Mitspracherechte, was ihre Bausubstanz angeht. Auch die kommunalen Bildungsdepartemente sind bei konkreten Baufragen wenig involviert. Ein systematischer Überblick über die Regelungen in den über 2250 Gemeinden ist mir nicht bekannt. In der Regel werden Aufträge im Schulhausbau, die ein gewisses Volumen erreichen, über Wettbewerbe ausgeschrieben. Die Wettbewerbsjury setzt sich in den meisten Fällen praktisch ausschließlich aus Architekten zusammen. Zumindest sind Architekten weit überproportional vertreten.

Ein Grund, weshalb die Bauaufgabe einseitig bei den Baudepartementen angesiedelt ist, dürfte sein, dass Schulbauten teilweise auch kommunale Funktionen als Gemeindehäuser übernehmen. Sie dienen als Wahl- und Stimmlokale, in kleineren Gemeinden als Gemeindesaal u. a. m.

In den letzten Dekaden wurden zahlreiche Schulreformen durchgeführt, die auch Einfluss auf den Raumbedarf und das Raumprogramm von Schulbauten haben. Die Schule differenziert sich zu einer hybriden multiprofessionellen Organisation aus. Dabei sind auch unterschiedliche Departemente mit verschiedenen Zuständigkeiten miteinander verzahnt. So sind neben dem Kernbetrieb des Unterrichtens, der immer noch in der Obhut der Kantone liegt, die Gemeinden für die neueren betrieblichen Institutionen wie die Schulleitungen, die Schulsozialarbeit und - wo vorhanden - die Tagesstrukturen zuständig. Diese Schulreformen verlangen nach baulichen Anpassungen und/oder Erweiterungen, die von den Gemeinden in Eigenregie umgesetzt werden.

Die Kantone sind für die Aufsicht und Regelung der Schulen zuständig. In Bezug auf den Schulhausbau legen sie Minimalanforderungen von Neu- und Umbauten fest, die der Sicherung des Unterrichts dienen (siehe EDKB 2015). Die Kantone sind vor allem über die Baudepartemente involviert, welche für die Erstellung der kantonalen Bauvorschriften und deren Durchsetzung zuständig sind. Zudem verfassen die kantonalen Erziehungsdirektionen Planungshilfen für die Gemeinden und betreiben in der Regel Beratungsangebote. ${ }^{3}$ Historisch gesehen sind die Planungshilfen in Form der ,Normalien“ entstanden (Gobat 1901;

3 Beispiele solcher kantonalen Planungshilfen sind: DVKL 2013; EDKB 2015; AfV 2004. 
Helfenberger 2013; Salvisberg 1870). Die Normalien sind Umsetzungsvorschläge für die Errichtung von Schulhäusern, da die Gemeinden, die in der Regel von Laien verwaltet wurden und werden, mit der Konzeption und Planung von Schulhäusern im 19. Jahrhundert finanziell und sachlich überfordert waren. Heute variieren die Vorgaben und Hilfen je nach Kanton beträchtlich. In der Regel umfassen die Planungshilfen Vorgaben über das Raumprogramm, die Raumvolumina und die Raumanforderungen. Zudem überprüfen die kantonalen Schulinspektionen die Einhaltung geltender Vorschriften.

Eine kantonale Instanz, die großen Einfluss auf den Möglichkeitsraum der baulichen Veränderungen hat, ist die Denkmalpflege. Deren Interessen stehen den pädagogischen bzw. schulischen Wünschen und Bedürfnissen oftmals diametral entgegen, wobei sich die Denkmalpflege in den meisten Fällen durchsetzt.

Die Eidgenossenschaft als oberste Instanz ist verpflichtende Auftraggeberin, die den Kantonen die Schaffung und den Betrieb der Schulen vorschreibt. Zudem erlässt sie bindende gesetzliche Verordnungen, etwa die Bestimmungen zu hindernisfreiem Bauen, die für alle öffentlichen Gebäude umgesetzt werden müssen. Als Besonderheit oder gar Anomalie wurde 1874 der Turnunterricht dem damals geschaffenen Eidgenössischen Militärdepartement - heute Eidgenössisches Departement für Verteidigung, Bevölkerungsschutz und Sport (VBS) - unterstellt. Dieses ist nach wie vor für die Konzeption und Inspektion des Schulturnens zuständig und legt die baulichen Mindestanforderungen von Sportanlagen und Sporthallen fest.

Die grundsätzliche Aufteilung der Zuständigkeiten zwischen Bund, Kantonen und Gemeinden ist seit der Entstehung der Volksschule stabil geblieben.

Neben den staatlichen Akteuren sind im Schulhausbau zahlreiche nichtstaatliche Akteure eingebunden. Für Architekten und Ingenieure sind es vor allem die umfangreichen Bautätigkeiten und das Bauvolumen, die für sie eine Beauftragung interessant machen. Schulhäuser entwickelten sich relativ rasch, spätestens „gegen Ende des 19. Jahrhunderts[,] zu einem relevanten Profilierungsfeld für Architekten“ (Helfenberger 2008: 307), das es bis heute geblieben ist. Historisch gesehen waren weitere Akteure bei der Entwicklung und dem Bau von Schulhäusern involviert: Im 19. und zu Beginn des 20. Jahrhunderts waren dies speziell die Schulärzte und Hygieniker. Die präventiv ausgerichtete Medizin nimmt von Beginn an die Schulen als ideale Orte der Implementierung von Gesundheitsförderung in den Fokus. Die Schulhygiene hatte einen großen Einfluss auf 
die Gestaltung der Schulhäuser, zum Beispiel in Form des typischen Hochparterres (siehe Hofmann 2008, 2016).

$\mathrm{Zu}$ nennen ist im Weiteren die Lehrerschaft, die als die eigentlichen ,Bewohner' der Gebäude betrachtet werden kann. Als generell schwach organisierte und wenig professionalisierte Berufsgruppe haben die Lehrer seit je keinen nennenswerten Einfluss auf die Konzeption von Schulbauten (vgl. Helfenberger 2013). Somit ist festzustellen, dass in den architekturrelevanten Debatten und Publikationen, die sich an Architekten und Ingenieure richten, kein genuin eigenes pädagogisches Programm zu konstatieren ist, wie dies parallel zu den reformpädagogischen oder auch zu den hygienisch-pädagogischen Diskursen existierte (Helfenberger 2008: 307 f.). Dieser Umstand stellt sich auch heute noch mehr oder weniger so dar, wenn die pädagogische oder erziehungswissenschaftliche Literatur gesichtet wird: Der Nichteinbezug des pädagogischen Personals in die Konzeption und Bauplanungen wird in der Literatur beklagt und das Feld als den Architekten überlassen dargestellt (siehe z. B. Braun et al. 2014; Forster \& Rittelmeyer 2010; Montag Stiftung Jugend und Gesellschaft Bonn 2011). Dieser Umstand bestätigt sich, wenn die Beurteilungsgremien der Architekturwettbewerbe betrachtet werden. In der Regel bestehen die Wettbewerbsjurys aus Architekt_innen, Vertretern/Vertreterinnen der Denkmalpflege (meist ebenfalls Personen aus der Architektur) sowie Vertretungen der kantonalen Erziehungsdirektionen, die fast immer auch aus Architekt_innen bestehen. Es ist also festzuhalten, dass die ,Bewohner', die den Auftrag der Gebäude funktional erfüllen, auf die Konzeption der Gebäude und der Schulanlagen faktisch keinen Einfluss haben.

\subsection{Zum Forschungsstand}

Zum Gegenstand Schulhausarchitektur ist eine überschaubare Anzahl von wissenschaftlichen Texten vorhanden, die für eine architektursoziologische Auslegung von Interesse sind. Es können vier Bereiche der vorhandenen Literatur unterschieden werden. Es sind dies zum Ersten die mehr oder weniger systematischen historischen Sammlungen und Dokumentationen von Schulbauten, zu denen auch Teile der entwicklungsgeschichtlichen und architekturhistorischen Publikationen zu rechnen sind. Zum Zweiten ist die Literatur der historischsoziologischen Bildungsforschung zu nennen, die der Architektur eine große 
Wirkung in der Ausdifferenzierung der Moderne zuschreibt und die sich sowohl mit den Gebäuden an sich als auch mit dem Thema Schulhausbau in seinen unterschiedlichen Facetten befasst. Zum Dritten kann die sozial- und erziehungswissenschaftliche und die psychologisch ausgerichtete Forschungsliteratur genannt werden, die die Architektur als Teil einer pädagogisch initiierten Anregung versteht. Als vierter Bereich ist eine Literaturgattung zu nennen, die vor allem normativer Natur ist und im Sinne entworfener Programmatiken auf die Gestaltung von Schulbauten Einfluss nehmen will. Im Folgenden wird ein kurzer Überblick über diese Bereiche gegeben.

\subsubsection{Der Schulhausbau in einer historisch-systematischen Perspektive}

Eine Reihe von empirischen Arbeiten sind als Sammlungen oder Kompendien zu betrachten, die jeweils einen meist regionalen und epochal organisierten Überblick über den Schulhausbestand umfassen. Die ältesten systematischen Sammlungen von Schweizer Schulhäusern liegen von Baudin $(1907,1917)$ vor. Neueren Datums sind entwicklungsgeschichtlich-architekturhistorische Abhandlungen über die Schulbauten im Kanton Luzern (Oberhänsli 1996), im Kanton Bern (Schneeberger 2005) und in der Stadt Bern (Gurtner 1997), im Kanton Waadt (Desroches et al. 2003; Heller \& Fornet 1997) und in Lausanne (Birke von Graevenitz et al. 2012). Eine Bestandsaufnahme der Schulhäuser der Stadt Zürich, die primär die architekturhistorischen Bezüge herausarbeitet, liegt von Dangel und Zangger (2008) vor. Diese systematischen Sammlungen sind vorwiegend deskriptiv angelegt.

Schneeberger (2005) geht der Frage nach, ob die Schulhäuser vor allem als Zweckbauten oder als Repräsentativbauten zu betrachten sind, und erstellt eine Übersicht über die Berner Bautypen im Schulbau des 19. Jahrhunderts. Auch Oberhänsli (1996) erarbeitet eine Typologie der Schulhäuser zwischen 1850 und 1950, wobei er diese als entwicklungsgeschichtliche Abfolge „,vom Eselstall zum Schulhauspavillon“ bezeichnet. Die Ausdifferenzierung von Schulbauten zu einem eigenen Gebäudetyp hat für Oberhänsli zur Folge, dass Schulen neben der Kirche zu den „bedeutenden Bauwerken im Ortsbild“ (1996: 74) werden. Oberhänsli unterscheidet neun Grundrisstypen, die keine festen Systeme bilden, sondern sich in einem stetigen Wandel befinden, der insgesamt als ein stufenweiser 
Fortschritt dargestellt wird: neue Architektur gehe jeweils mit einer neuen Pädagogik einher. Auch Dangel und Zangger (2008) halten fest, dass die vorhandene Literatur die Geschichte des Schulhausbaus ausschließlich als stufenweise Fortschrittsgeschichte deutet. Zudem stellen sie zwei zusammenhängende Merkmale des Schulhausbaus fest: Die Architektur von Schulbauten ist sowohl architektonisch als auch von der Bausubstanz her von überdurchschnittlicher Qualität. Dies hat zur Folge, dass die Interessen der Schulen denjenigen der Denkmalpflege überaus häufig gegenüberstehen und mit ihnen kollidieren.

\subsubsection{Soziologische Perspektiven auf Schulbauten}

Göhlich (1993, 2009) untersucht die historische Entwicklung von Schulbauten vom Mittelalter bis in die Gegenwart. Er stellt drei Tendenzen fest, die sich in der Entwicklung der Schulhausarchitektur herauskristallisieren: die Entwicklung des Schulraums zur Heimat, die Entwicklung des Schulraums zur Lernlandschaft und die Entwicklung des Klassenzimmers zu einem dezentral organisierten Raum.

Knutti-Baumann stellt im föderalistisch-subsidiären System einen verborgenen Zentralismus im Schulgeist fest. Nach ihm ist die „Geschichte des Schulhausbaus in der Schweiz (...) primär eine Geschichte der Architektur, die den Fokus auf das Schulgebäude selbst, dessen architektonische und stilistische Entwicklung und Typologie sowie auf die Problemlösungsstrategie der Architekten bei der Umsetzung pragmatischer und ästhetischer Bedürfnisse richtet“ (KnuttiBaumann 1997: 357). Dabei orientieren sich die Architekten fast ausschließlich an der Reformpädagogik. Diesen Eindruck teilt auch Helfenberger (Helfenberger 2008, 2013), die den Forschungsfokus auf die normativen Debatten legt, die den Schulhausbau begleiten. Sie stellt die Geschichte des Schulhausbaus als eine Normengeschichte dar, die von medizinisch-hygienischen Normen des 19. Jahrhunderts hin zu reformpädagogischen Konzepten in Verknüpfung mit architektonischen Entwicklungen steht. Die Debatten über Schulhausarchitektur gründen auf einem Prozess der Normendefinition. Dieser wird von den Perspektiven, Interessen, Motivationen und Zielen der involvierten Akteure sowie deren Programmen geprägt (Helfenberger 2013: 13). Pädagogische Überlegungen spielen dabei keine oder eine untergeordnete Rolle. Die Debatte wird vor allem von den 
Bauplanern, den Behörden und später von den sich in Berufsverbänden organisierenden Architekten bestimmt. Dies hat zur Folge, dass der architektonische ,Wert' eines Gebäudes zugleich den pädagogischen ,Wert' bestimmt, ohne dass Letzterer ausbuchstabiert wird oder ausbuchstabiert werden müsste. Man kann das Ergebnis daraufhin zuspitzen, dass ,gute Architektur' gleichsam als ,gute pädagogische Architektur' angesehen wird. Die Fallrekonstruktionen im empirischen Teil verweisen darauf, dass diese Deutungen auch heute noch Bestand haben.

Der Ärztestand ist ein weiterer Akteur, der die Schule und die Schulhausarchitektur im ausgehenden 19. Jahrhundert maßgeblich mitprägte. Die Ärzte und Hygieniker nutzten die Schule für die Vermittlung von gesundheitsförderlichem Verhalten und gesundheitsrelevantem Wissen. Dies äußert sich unter anderem darin, dass sich innerhalb der Gesundheitsbewegung ein eigenes Berufsfeld der Schulhygieniker ausdifferenzierte (vgl. dazu Hofmann 2008, 2016; Imboden 2003), das in Form der Schulärzte bis heute besteht. Die Schulhygiene bestimmte und entwickelte tonangebend das Schulmobiliar, aber auch einige der bautechnischen Merkmale von Schulhäusern, die die Formensprache des Gebäudetyps Schulhaus maßgeblich geprägt haben: zum Beispiel das Hochparterre. Aber auch die Vorschriften, dass die Fensterfronten mindestens ein Viertel der Bodenfläche betragen müssen, hat die Schulhausarchitektur mitbestimmt. Hofmann stellt fest, dass ,die hygienische Forderung nach ,Licht und Luft' (...) die Schulhausarchitektur seit dem ausgehenden 19. Jahrhundert in hohem Maße (bestimmte). Es wurden neuartige Grundrisslösungen entwickelt, die bis weit ins 20. Jahrhundert hinein für den Schulhausbau maßgebend blieben“ (Hofmann 2016: 78).

Eine ganze Reihe sozialwissenschaftlicher Diskursanalysen von Foucault oder in der Tradition von Foucault betrachten Schulgebäude unter einem machttheoretischen Blick (Caruso 2003; Foucault 2012b, 2012a; Kupfer 2011). Der Architektur wird ein entscheidendes Moment in der Herstellung eines Dispositivs zugeschrieben, das für die Macht- und Herrschaftsausübung der Moderne charakteristisch ist: die Transformation von der Beherrschung des Körpers zur Beherrschung der Seele. Gebäude wirken unter Umständen schon durch ihre Monumentalität disziplinierend, indem sie Beklemmung auslösen und auf Kontrolle gerichtet sind (siehe Rieger-Ladich \& Ricken 2009). Für Foucault ist wesentlich, wie die Subjekte über die Architektur dazu gebracht werden, sich selbst zu führen: 
„Die Organisation eines seriellen Raumes ist eine der großen technischen Mutationen des Elementarunterrichts, der das traditionelle System (...) abgelöst hat. Indem er individuelle Plätze zuwies, hat er die Kontrolle eines jeden und die gleichzeitige Arbeit aller möglich gemacht. Er hat eine neue Ökonomie der Lernzeit organisiert. Er hat den Schulraum zu einer Lernmaschine umgebaut - aber auch zu einer Überwachungs-, Hierarchisierungs-, Belohnungsmaschine.“ (Foucault 2012b: 188)

Damit räumliche Anordnungen eine Wirkung entfalten, reicht es nicht, räumliche Strukturen zu schaffen, die die Gleichzeitigkeit erlauben. Architekturen müssen so auf die Subjekte einwirken, dass sich diese konform und diszipliniert verhalten und autonom aus sich heraus denjenigen Tätigkeiten nachgehen, die von ihnen verlangt werden.

„Die Herstellung von Disziplin erfolgt über die Architektur der Schule, sie ist räumlich in voneinander abgesonderten Klassenzimmern und durch die Form der Anordnung von Tischen und Stühlen aufgeteilt. Im Gegenüber von Schulklasse und Lehrperson werden Interaktionsformen strukturiert, die einer strengen Dramaturgie unterliegen: Räume und Zeiten für offene Interaktionen und Begegnungen gibt es in der traditionellen Schule fast nur vor und nach dem Unterricht sowie in den Pausen." (Giddens 1988: 188)

Räumliche Dispositionen sind Teil eines ausgeklügelten Dispositivs, die in ihren räumlich-territorialen Anordnungen auf Disziplinierung ausgelegt sind (Caruso 2003), zum Beispiel stellen schmale Korridore eine Verengung von Interaktionsmöglichkeiten dar. Als Disziplinierungstechniken sind besonders auch das Schulmobiliar, die sogenannten Subsellien, zu sehen. Hnilica sieht die Schulbank als „Kristallisationspunkt einer disziplinierenden Schularchitektur“ (2010: 141). Über sie wird auf die Geometrie der Sitz- und Körperhaltungen eingewirkt, wobei das Ziel nicht lediglich gesundheitsbezogene Körperformationen sind. Die Schulbank ist vielmehr ein technischer Apparat, mit dem über den Körper auf den ,Geist" eingewirkt wird (zu den Subsellien siehe auch Heller \& Jost 1988).

Eine Reihe von gegenwärtigen Untersuchungen, die einen mehr oder weniger starken Bezug zur Heterotopologie Foucaults (1991, 2013) haben, kommen zum Schluss, dass Schulhäuser beziehungsweise Schulen Orte darstellen, welche die gesellschaftlichen Strukturen teilweise außer Kraft setzen, wenden, spiegeln oder auf eigene stark normativ konnotierte oder auch imaginierte Realitäten richten. Pädagogische Räume sind Gegen-, Schutz- und/oder Entfaltungsräume. Sie sind Räume von „Paradiesentwürfen“ (Baader 1999), denen ein zukünftiger normativer Bezug und unter Umständen utopische Entwürfe inhärent sind. Herrmann (2013) und Böhme, Flasche und Herrmann (2016) sehen in Schular- 
chitekturen Heterotopien. Dabei sind diese weniger auf einen utopischen Entwurf, sondern vielmehr auf die Herstellung eines ,pädagogischen Machtraums“ (Böhme \& Herrmann 2011) gerichtet. Dieser Machtraum strukturiert die Interaktionsmöglichkeiten, die im Rahmen Schule gegeben sind. Die Schule schafft einen Machtraum, der über die Schule hinaus wirkt.

\subsubsection{Der Raum als Erzieher - Erziehungswissenschaftliche Standpunkte}

Auch die erziehungswissenschaftliche und teilweise psychologisch ausgerichtete Literatur, die sich mit Schulbauten befasst, sieht in der Architektur ein wesentliches und praxisprägendes Element der Schule (eine Übersicht gibt Nugel 2014). Ausgangspunkt sind oft anthropologische Annahmen, die sich auf Bollnow beziehen. Für Bildung relevant ist der erlebte Raum, der immer in Bezug zum sich bewegenden Menschen steht (Bollnow 1960, 1971). Der Raum und das räumliche Setting wird unter dem Aspekt der Interaktion mit der Umgebung gesehen, wobei dem Raum eine Funktion als „,drittem Erzieher“ (Brüschweiler \& Reutlinger 2014; Schäfer \& Schäfer 2009) zugeschrieben wird. Der Raum oder die Architektur wird hier jedoch nicht unter der disziplinierenden Perspektive, sondern als Herstellung von Anregung und daher als erstellte oder nach pädagogischen Kriterien zu erstellende Lernumgebung gesehen. Der Raum ist Teil der sozialisatorischen Aneignung der Welt (Rittelmeyer 2002), als gestalteter und pädagogisch strukturierter Raum ist er ein ,Lernraum“, ,Bildungsraum“ und ,Erziehungsraum‘. Die Umgebung wird als Ermöglichungsraum gesehen, in dem die Kinder sich selbsttätig und intrinsisch motiviert bewegen (z. B. Hentig 1975, 1997). Als Ermöglichungsraum wird der Schulraum als nach pädagogischen Kriterien zu strukturierender Raum gesehen. Der pädagogische Bezug wird dabei oft in unterschiedlichen reformpädagogischen Konzepten ausgemacht. Perlick (1969: 27 ff.) zeigt auf, wie der „Schulraum zu einem Lehrmittel“ wird. Er kommt zum Schluss, dass „,der Raum, den eine Schulklasse für die vielfältigen pädagogischen Situationen braucht, ein Gruppenarbeitsraum [ist], der durch Form und Einrichtung der Entfaltung unterrichtlichen Geschehens jede Freiheit lässt“" (Perlick 1969: 90). Die Schule als größerer Rahmen hat überschaubare, funktional differente Bereiche herzustellen, und nach außen soll die Schule eine Beziehung mit der Nachbarschaft eingehen (ebd.). 
Göhlich (1992, 1997a) befasst sich mit den unterschiedlichen reformpädagogischen Konzepten - hauptsächlich der Reggiopädagogik und dem Konzept des offenen Unterrichts -, die zur ,Neuen Reformpädagogik“ fusionieren. Die Neue Reformpädagogik ist nicht mehr anthropologisch, sondern sozialisationstheoretisch fundiert. Sie orientiert sich stark non-personal, wobei das Kind das Materialgebende und Interessenformulierende ist. Im Gegensatz zur klassischen Reformpädagogik, welche die Natur als pädagogisch wertvolle Umgebung betrachtet, ist dies bei der Neuen Reformpädagogik die Kultur. Dementsprechend wird die pädagogische Umgebung als anregende Umgebung erschaffen (Göhlich 1997b: 20 ff.).

Diesem Gedanken folgen viele neuere Publikationen. Watschinger und Kühebacher (2007) fordern Lernwelten, die den neuen Anforderungen der Wissensgesellschaft und deren Wissensformen gerecht werden. Sie fordern eine stärkere Autonomie der Schulen, die sich nicht nur in der unterrichtlichen und pädagogischen Schulentwicklung, sondern auch räumlich und architektonisch selbst entwickeln sollen. Trotzdem stellen sie innerhalb dieser autonomen Schulentwicklung Leitlinien auf und fordern als wesentliches Mittel die Setzung von Standards: Zentral ist die „Zusammengehörigkeit von unterrichtlicher und erzieherischer Arbeit“" (Watschinger \& Kühebacher 2007: 22): Unterricht und Raumprogramm soll auf Individualisierung und Differenzierung gerichtet sein, und Lehrpersonen sollen ihr Berufsverständnis ändern. Sie sollen nicht mehr instruieren, sondern vor allem Arbeitsprozesse arrangieren, wofür sie passende - vorstrukturierte oder arrangierte - Architekturen und Räumlichkeiten brauchen.

Die erziehungswissenschaftliche Literatur ist insgesamt wenig empirisch fundiert. Sie befasst sich stark mit der normativen Frage, was eine gute Schule ist.

\subsubsection{Schularchitektur als Vehikel der Schulentwicklung - Schulpädagogische Positionen}

Eine Reihe von Arbeiten stellt explizit Forderungen an die spezifische Beschaffenheit und Funktionalität der Architektur von Schulgebäuden. Neue pädagogische Konzepte und neue Lernformen sollen in die Architektur einfließen. In diesem Sinne sind es keine empirischen Forschungsergebnisse - allenfalls mit 
best practice-Beispielen illustriert -, sondern vor allem Forderungen und Vorschläge, die sich als Impulsgeber aus pädagogischer Sicht für Architekten und Baubehörde verstehen. Sie nehmen in Anspruch, Antworten auf die normativen Fragen zu liefern, wie eine gute, zeitgemäße Schule auszusehen hat. Sie sollen hier kurz angesprochen werden, da sie maßgeblich auch den erziehungswissenschaftlichen Diskurs mitprägen.

Die Montag Stiftung setzt sich seit Jahren für einen zukunftsfähigen Schulbau ein. In ihrem Selbstverständnis sind dies Schulen, die nach außen eine Öffnung zur Stadtgemeinschaft realisieren und die nach innen spezifisch auf eine zeitgemäße Pädagogik gerichtet sind. Zeitgemäßer Unterricht beinhaltet im Wesentlichen die Inklusion und den Umbau der Schulen zu Lern- und Bildungslandschaften (Montag Stiftung Jugend und Gesellschaft Bonn 2011). In eine ähnliche Richtung zieht auch die Stiftung Wüstenrot (Jocher et al. 2011). Beide nehmen für sich in Anspruch, die Schulen ,radikal“ neu zu denken. Kern davon ist die Konzipierung von Schulhäusern als sogenannte Bildungs- und Lernlandschaften, in denen sich die Schüler_innen weitgehend selbstständig und selbsttätig den Schulstoff und andere Kompetenzen aneignen. Diese Konzepte gehen oft mit pädagogischen Konzepten der Individualisierung von Unterricht und Lerninhalten in einem selbst organisierten und selbst gesteuerten Lernen u. a. m. einher.

Braun et al. (2014) fordern ausdrücklich einen flexiblen und multifunktionalen Schulbau, um Funktionskonflikte zwischen Mehrfachnutzungen zu minimieren. Sie fordern Schulen als entgrenzte Lernorte, wo neue Räumlichkeiten neue Lernkulturen formen werden. Kerngedanke ist die Einbindung neuer Funktionen: Kindergärten sollen stärker in die Schulhäuser integriert werden; Schulen sollen als integrative Schulen auch die bisherigen Sonderschulen integrieren, und last but not least sollen Tagesstrukturen räumlich-örtlich Teil der Schulen sein. Die dergestalt zu konzipierenden Schulen sollen als „SpielRäume“ (Braun \& Fachhochschule 2015) fungieren, wobei auch den Lehrpersonen eine Rolle zugewiesen wird, die weniger der Wissensvermittlung dient, als vielmehr derjenigen eines Coachs entspricht. Die Anregung durch Raum und Artefakte steht im Zentrum, und die pädagogischen Interaktionen zwischen Lehrperson und Schüler_innen(-Gruppen) kommen im Bedarfsfall steuernd oder ergänzend dazu.

Es kann die etwas grobschlächtige Schlussfolgerung gezogen werden, dass in der Forschungsliteratur, namentlich in den disziplinären Linien Soziologie und Erziehungswissenschaften, zwei grundsätzlich differente Perspektiven dominie- 
ren. In die Theoriesprache der Objektiven Hermeneutik übersetzt, kann man sagen, dass die soziologischen Studien Schulhäuser unter dem Aspekt von disziplinierenden Routinen betrachten. Sie richten den Fokus darauf, wie Wissensformationen über Herrschaft von den Subjekten internalisiert werden. Die erziehungswissenschaftliche Literatur hingegen legt den Schwerpunkt auf die krisenevozierende Wirkung der Architektur. Schulhäuser und Räume werden als pädagogische Instrumente und Anregungen verstanden. Dabei dominieren oft normativ konnotierte Konzepte, wobei die empirische Betrachtung häufig fehlt. Denn es kann vermutet werden, dass die Architektur beziehungsweise die Umgebung nur einer von vielen Faktoren ist, die einen Einfluss auf Unterricht haben. Es ist doch anzunehmen, dass auch in den vielversprechenden Lernlandschaften ein schlechter Unterricht vonstattengehen kann, während umgekehrt auch in scheinbar ungeeigneten Schulhäusern hervorragender Unterricht möglich ist. Oder andersrum gefragt: Kann eine gute Architektur guten Unterricht garantieren?

Die Anlage dieser Arbeit kann darauf höchstens indirekt eine Antwort geben. Ausgangslage ist, dass Architekturen eine Einflussstruktur darstellen, die einen Möglichkeitsraum vorstrukturieren. Der Fokus dieser Arbeit ist auf die empirische Rekonstruktion gerichtet, welche Anregungen durch Architektur initiiert werden. Im Blick sind die Rekonstruktion der Bedeutungsstrukturen und Handlungsdispositionen, die den sogenannten Bildungslandschaften inhärent sind und die einem autonomen Bildungsprozess, gewissen (schul)pädagogischen Konzepten und Unterrichtsformen unter Umständen eher hinderlich entgegenstehen können. Die Analysen sind aber keine Aussagen über die faktisch vollzogenen Handlungen. Den Analysen liegt aber die Annahme zugrunde, dass es gewisse Passungsverhältnisse zwischen pädagogischen Konzepten und räumlichen (An-)Ordnungen gibt.

Open Access Dieses Kapitel wird unter der Creative Commons Namensnennung 4.0 International Lizenz (http://creativecommons.org/licenses/by/4.0/deed.de) veröffentlicht, welche die Nutzung, Vervielfältigung, Bearbeitung, Verbreitung und Wiedergabe in jeglichem Medium und Format erlaubt, sofern Sie den/die ursprünglichen Autor(en) und die Quelle ordnungsgemäß nennen, einen Link zur Creative Commons Lizenz beifügen und angeben, ob Änderungen vorgenommen wurden.

Die in diesem Kapitel enthaltenen Bilder und sonstiges Drittmaterial unterliegen ebenfalls der genannten Creative Commons Lizenz, sofern sich aus der Abbildungslegende nichts anderes ergibt. Sofern das betreffende Material nicht unter der genannten Creative Commons Lizenz steht und die betreffende Handlung nicht nach gesetzlichen Vorschriften erlaubt ist, ist für die oben aufgeführten Weiterverwendungen des Materials die Einwilligung des jeweiligen Rechteinhabers einzuholen.

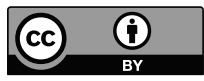

